\title{
Visualizations of Mistral and Tri Alpha Plasmas
}

\author{
Greg Foss \\ Texas Advanced Computing Center \\ The University of Texas at Austin \\ Austin, TX, USA \\ foss@tacc.utexas.edu
}

\author{
Wendell Horton \\ Institute for Fusion Studies \\ The University of Texas at Austin \\ Austin, Texas, USA \\ horton@physics.utexas.edu
}

\author{
Lee Leonard \\ Institute for Fusion Studies \\ The University of Texas at Austin \\ Austin, TX, USA \\ cloud@utexas.edu
}

\begin{abstract}
Plasma expert Wendell Horton from the Institute for Fusion Studies at the University of Texas at Austin is running simulations on the Stampede2 supercomputer at the Texas Advanced Computing Center (TACC) to investigate plasma turbulence characteristics to validate design parameters at the ITER (International Thermonuclear Experimental Reactor, and Latin for "the way") tokamak project in southern France. Visualizations show waves from simulated electromagnetic fields corresponding to those generated in the lining of the magnetic device as the plasma inside creates fusion energy.
\end{abstract}

\section{CCS CONCEPTS}

- Human-centered computing $\rightarrow$ Scientific visualization;

\section{KEYWORDS}

plasma simulation, fusion studies, visualization, Mistral simulation,TriAlpha simulation

\section{ACM Reference Format:}

Greg Foss, Wendell Horton, and Lee Leonard. 2018. Visualizations of Mistral and Tri Alpha Plasmas. In PEARC '18: Practice and Experience in Advanced Research Computing, fuly 22-26, 2018, Pittsburgh, PA, USA. ACM, New York, NY, USA, 2 pages. https://doi.org/10.1145/3219104.3229430

\section{INTRODUCTION}

The nonlinear dynamics of fluids, and even more so of plasmas, creates complex 3D structures. Plasmas are composed of heavy ions and light-weight,fast moving electrons creating two different fluid components. The ion fluid carries the mass of the fluid, and fast moving electron fluid creates the dominant electric fields and currents. The slower moving ion carries the mass of the plasma, and the fast moving electron fluid creates the electromagnetic fields. The electron motion is faster and different from that of the ions. The Hall semiconductor and E- layer of the ionosphere are two extreme examples where the ions move very slowly and the electrons respond freely to small electric and magnetic fields. Thus, the 3D simulations of the nonlinear dynamics of the electron and ion fluid components are essential in order to see and understand the distinct motions of the two fluids. In the plasma text books this is called the two-component fluid model but full complexity of this motion is revealed with $3 \mathrm{D}$ visualizations.

Permission to make digital or hard copies of part or all of this work for personal or classroom use is granted without fee provided that copies are not made or distributed for profit or commercial advantage and that copies bear this notice and the full citation on the first page. Copyrights for third-party components of this work must be honored.

For all other uses, contact the owner/author(s).

PEARC '18, fuly 22-26, 2018, Pittsburgh, PA, USA

(c) 2018 Copyright held by the owner/author(s).

ACM ISBN 978-1-4503-6446-1/18/07.

https://doi.org/10.1145/3219104.3229430

\section{VISUALIZATION}

ParaView [1] software was used to generate volume renderings of four scalars: electic potential, magnetic flux, plasma density, and plasma pressure, from data generated on Stampede2 at TACC, in a series of 300-900 timestep simulations. Visualized is complex dynamics of the nonlinear Alfven waves with their related distortions of plasma density and pressure. Simulations show the nonlinear Alfven wave flux ropes in electric potential and magnetic flux fields, which produce the forces and waves we then see in plasma density and pressure. Dr. Horton is interested in comparing plasma waves in models from the TriAlpha (Figure 1) and Mistral (Figure 2) simulations.

\section{SCIENCE}

Some of the key equations and theories that pertain to the visualizations are described briefly here, but a detailed description of the mathematical and theoretical underpinnings represented in this project are beyond the scope of this paper. Please refer to Dr. Horton's publications ITER Physics [Horton-2015] and Turbulent Transport in Magnetized Plasmas [Horton-2012] for the full story.

In the TriAlpha simulations of the four interacting fields we kept the most dominant nonlinearity for the plasma that is the same mathematical structure as in neutral fluids and gasses. This is the convective derivative of the velocity field in the $\mathrm{F}=$ ma motion of the mass density of the plasma which is almost entirely from the ions. The electrons mass is tiny compared to that of the ions but the electrons carry the electric current which make the dominant force $\mathrm{F}$ on the plasma. In designing plasma confinement the first step is to take the forces from gradient of the fluid pressure and balance them with electromagnetic force from electron current vector crossed into the magnetic field vector.

Simulations with the Mistral fusion model showed the coherent rotational motion and some lower order modes called $m=1, m=2$ in the literature of the plasma column with very coherent nonlinear structure. The nonlinearities are labeled as SNL111. Other nonlinearities are labeled with SNL-ijk for the dynamics in the i field from the interaction of fields $\mathrm{j}$ and $\mathrm{k}$. This allows one to inquire what is the roll of a particular nonlinear interaction by varying the value of strength of each nonlinear term given by SNL-ijk.

In addition more recent simulations of a similar plasma we included two more nonlinear terms labeled SNL122 and SNL 212 that transfer energy back and forth between the kinetic energy of the plasma and the magnetic energy of the plasma.

The movies from the Mistral runs (Figure 2) show the 3D complex structures of Alfven waves developing into what are called flux ropes. These flux ropes are detected by satellites at the Lagrangian-1 point where the pull of gravity of the Earth is balanced by the pull 


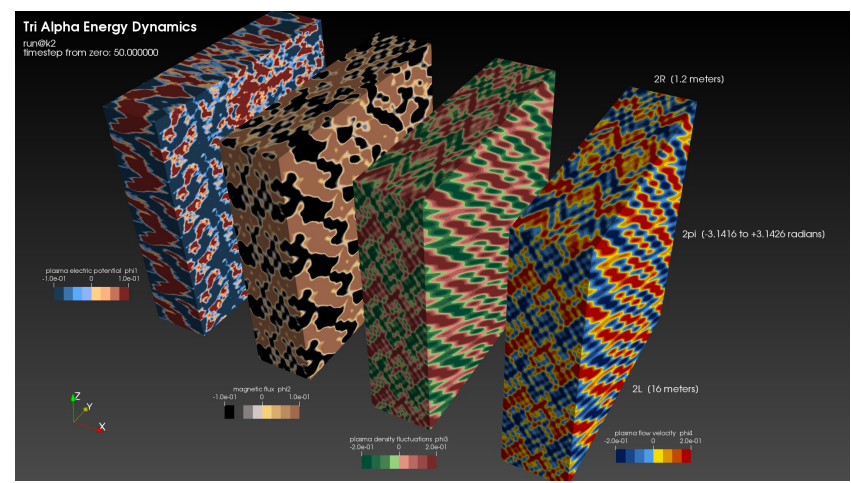

Figure 1: Left to right are TriAlpha simulation data fields Electric Potential(V), Magnetic Flux(Wb), Plasma Density $\left(1 / \mathrm{m}^{3}\right)$, and Plasma Pressure(Pa). Visualization by G. Foss, TACC

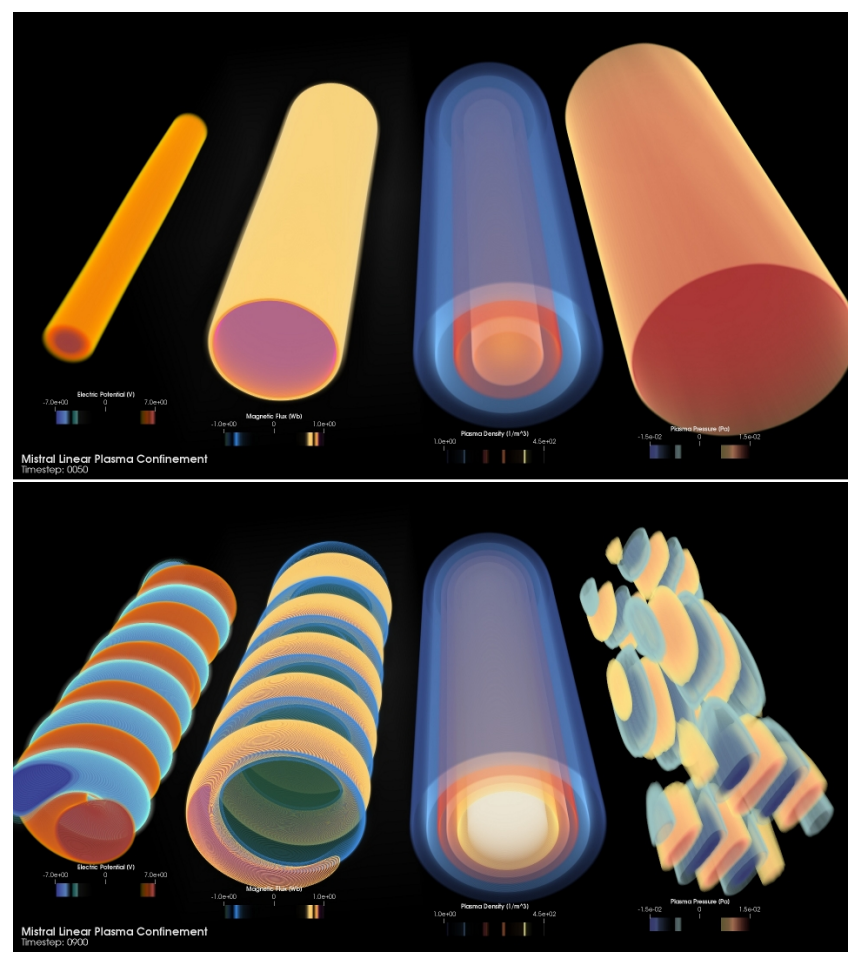

Figure 2: Timesteps from beginning (top) and end (bottom) of Mistral simulation data. Left to right are Electric Potential(V), Magnetic Flux(Wb), Plasma Density $\left(1 / \mathrm{m}^{3}\right)$, and Plasma Pressure(Pa). Visualization by G. Foss, TACC

of gravity from the Sun. The US has satellites that permanently hover at the Lagrangian-1 measuring these nonlinear waves as they come to the Earth. There is about a 50 minute warning time from the L1 point spacecraft data to the time these structures hit and disrupt the communication systems on the Earth. Clearly, the accurate detection and forecast of the strength of these solar storms and substorms is critical to the high technology societies on Earth.
The storms are also of interest as they strike Jupiter, Mars and other planets, but the forecasting on Earth has large defense and economic value.

\section{CONCLUSION}

The simulations are still in an academic frame showing the basic plasma physics but have the potential to develop with the petascale computers and with AI algorithms. Knowledge of a coming solar storm- that is forecasting ability even with less than a one hour alert- has large economic, health and defense value to a country.

\section{ACKNOWLEDGMENTS}

W. Horton's research is funded in part by US Department of Energy grant DE-FG02-04ER54742.

Much thanks to Anne Bowen of the TACC Scalable Visualization Technologies group for her very helpful technical support.

\section{REFERENCES}

[1] https://www.paraview.org/

[Horton-2012] Horton, W., 2012, Turbulent Transport in Magnetized Plasmas, 2nd Edition, World Scientific.

[Horton-2015] Horton, W., Benkadda, S., 2015, ITER Physics, World Scientific.

Received May 2018 